\title{
Self-optimised vision correction with adaptive spectacle lenses in developing countries
}

\author{
M. G. Douali and J. D. Silver* \\ Department of Atomic and Laser Physics, University of Oxford, Clarendon Laboratory, Parks Road, \\ Oxford OX1 3PU, UK
}

\begin{abstract}
It is estimated by the World Health Organization that about 1 billion people in the developing world would benefit immediately from distance vision and near vision correction if it were available to them. Here we address this problem and provide a solution by correcting vision in the field with adaptive liquid-filled variable focus lenses. We describe the details of the lens and present the results of a series of experiments performed in Ghana, Nepal, Malawi and South Africa that demonstrate that it is possible by simple means to self-determine and obtain correct refraction using such lenses.
\end{abstract}

Keywords: adaptive eyecare, Adspecs, ametropia, liquid-filled lens, spectacles, variable focus

\section{Introduction}

Nature's prototype eye may well have consisted of a drop of liquid converging rays of light onto photosensitive cells. The optical properties of such a liquid drop are clear when observing the printed word through a drop of water spilt on a page. The refractive power and optical quality of the curved surface of a liquid confined by an elastic membrane are the properties utilised in this study to correct refractive error in the human eye. Given its apparent geometric beauty and perfection the eye still suffers from a number of sometimes quite serious optical defects, the most important and most common of these being spherical ametropia, or defocus. Not only is defocus the most important, but also the most easily corrected; at least for those who have access to a trained optometrist and a selection of solid lenses.

For nearly 1 billion people in the developing world currently without the vision correction they require

\section{Received: 8 July 2003}

Revised form: 27 February 2004

Accepted form: 4 March 2004

*JDS has an association with Adaptive Eyecare Ltd, a UK company, the principal mission of which is to bring affordable vision correction to developing world countries.

Correspondence and reprint requests to: Prof. J. Silver. Tel.: + 44 (0) 1865 272206; Fax: + 44 (0) 1865272400.

E-mail address: josh.silver@new.oxford.ac.uk
(World Health Organization, 1987), the benefits of providing a simple and inexpensive means of correcting refractive error are clear, as poor vision can hinder a child's educational development and an adult's productivity at work. Studies have demonstrated the prevalence of visual impairment amongst children. A study performed in Shunyi District, China, by Zhao et al. (2000) sampling a total of 6134 children revealed that by the age of 15 years $46 \%$ of children were myopic $(>-0.50 \mathrm{D})$ and that the prevalence of uncorrected vision in at least one eye was $13 \%$. It also showed that refractive error was the cause in $89 \%$ of the 1236 eyes with reduced vision. The results from a study in La Florida, Chile, by Maul et al. (2000) indicated that more than $7 \%$ of school-age children could benefit from the provision of proper spectacles. It concluded that efforts are needed to make existing programmes that provide free spectacles to schoolchildren more effective. Another study in India by Dandona et al. (2002) established that, for children between the ages of 7 and 15 years, $70 \%$ of those who had visual acuity of $20 /$ 40 or worse would benefit from spectacles. The study recommended that effective strategies be developed to eliminate this easily treated cause of visual impairment.

To give a broader indication of the proportion of adults who need vision correction for ametropia an average can be found from the results published by the surveys of Strömberg (1936), Stenström (1946), and Sorsby et al. (1960). A total of 8187 eyes of adults 
under 35 years of age were tested and $67 \%$ required correction for ametropia, defined, in these studies, by the need of a correction of magnitude greater than $1 \mathrm{D}$. Another study in the US by Sperduto et al. (1983) using results from the 1971-72 National Health and Nutrition Examination Survey showed that the prevalence of myopia among persons between 12 and 54 years was over $24 \%$. A comprehensive Ministry of Health survey in the UK in 1962 (Bennett and Rabbetts, 1998) of the distance prescriptions made showed that $91 \%$ were in the range -6 to $+4 \mathrm{D}$. Therefore by using a lens with a variable power between -6 and $+6 \mathrm{D}$ the vast majority of prescription refractions could be achieved. If the lens can only correct spherical ametropia its usefulness would not be greatly reduced as demonstrated by the same survey which showed that of the prescription spectacle-wearing population a very large proportion $(84 \%)$ required a cylinder correction of $<1 \mathrm{D}$.

\section{The Adspec lens}

We developed the Adspec lens to provide a simple, effective and affordable means of vision correction whereby it is possible for the wearer to adjust the refractive power of each lens independently to optimise his or her refraction. This would make vision correction accessible to those in areas of the world where there is either a lack of professionally trained optometrists or where the cost of traditional spectacle lenses and professional consultation is prohibitively expensive.

For a lens to be suitable for correcting human vision the optical quality of the lens must be at or above that of the human eye itself if it is not to introduce additional aberrations reducing image quality. For the case of monochromatic light, the wave aberrations of the eye have been characterised by Porter et al. (2001) and Guirao et al. (2002) who showed that for a $5.7 \mathrm{~mm}$ pupil the total rms wave-front error arising from contributions other than defocus was approximately $0.9 \mu \mathrm{m}$. Using this criterion as a guide we have designed a variable power spherical lens which can be mounted in a spectacle frame. Two thin membranes $23 \mu \mathrm{m}$ thick are sealed at a circular perimeter of diameter $42 \mathrm{~mm}$ and secured by a frame. The volume enclosed by the membranes is filled with a liquid of refractive index 1.579. The optical power of the resulting lens is determined by the curvature of its surfaces and this is controlled by varying the volume of liquid in the lens. An interferometer experiment performed on a stretched sheet of the membrane film showed that the film thickness varied by less than about $\pm 0.4 \mu \mathrm{m}$ per $10 \mathrm{~mm}$ linear displacement across its surface. This ensured that the above wave-front error would not be exceeded - in fact the wave-front error introduced by the

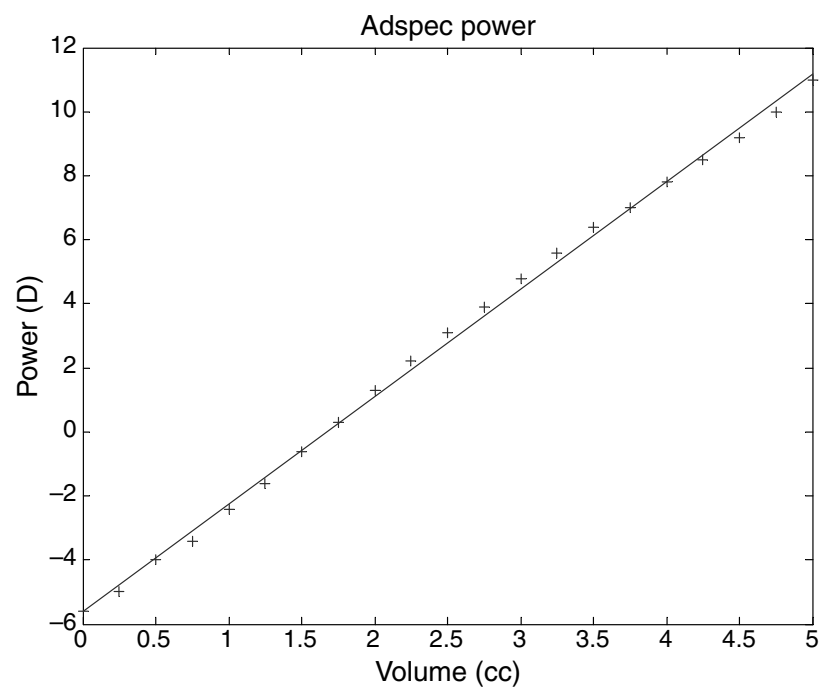

Figure 1. The experimentally determined refractive power (D) of the Adspec lens as a function of the lens volume $\left(\mathrm{cm}^{3}\right)$. The linear regression line has a slope of $3.60 \pm 0.04 \mathrm{D} \mathrm{cm}^{-3}$. This compares well with the value of $3.79 \pm 0.30 \mathrm{D} \mathrm{cm}^{-3}$ predicted by Equation (1).

membrane is calculated to be approximately only one half of that produced by the average eye.

A mathematical analysis of the lens surface is possible and the relationship between the refractive power of the lens and the liquid volume can be derived. ${ }^{1}$ The deformation of the elastic membrane produces a parabolic surface. If the effects of gravity are neglected and the deflection of the membrane surface is small compared with its radius of curvature, then to a first approximation, the surface of the lens is spherical and its refractive power, $P_{\text {Adspecs }}(\mathrm{D})$, is given by

$P_{\text {Adspecs }}=\frac{64\left(n_{\mathrm{L}}-1\right)}{\pi d^{4}}\left(V-V_{0}\right)$,

where $V$ is the actual volume of liquid contained by the lens $\left(\mathrm{m}^{3}\right), V_{0}$ is the residual cavity volume $\left(\mathrm{m}^{3}\right)$ and $d$ is the diameter of the lens $(\mathrm{m})$. This is in good agreement with the results of the experimental determination of the power-volume relationship shown in Figure 1. The linear regression line (solid) has a slope of $3.60 \pm 0.04 \mathrm{D} \mathrm{cm}^{-3}$, which compares well with the value of $3.79 \pm 0.30 \mathrm{D} \mathrm{cm}^{-3}$ predicted by Equation (1). Gravitational effects can also be accounted for; Sugiura and Morita (1993) showed that the additional aberrations are negligibly small compared with standard retail spectacle lenses. The off-axis performance of the Adspec lens is similar to any thin bi-spherical solid lens.

Two such identical lenses are then mounted in a specialised spectacle frame to form variable focus

\footnotetext{
${ }^{1}$ The details of the derivation of this formula and formulae describing the surface of the lens are included in Appendix A.
} 


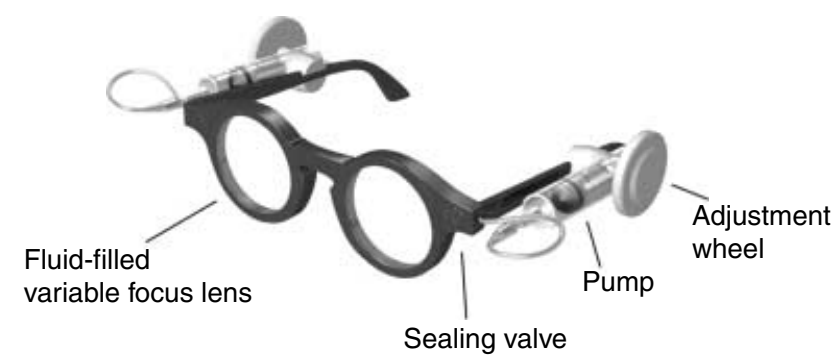

Figure 2. The Adspecs complete with detachable adjustment wheels and pumps. Once the optimum power setting has been obtained by the wearer the valves are sealed and the pumps are removed (Figure reproduced by kind permission of D. Crosby).

adaptive spectacles, or Adspecs, and are shown in Figure 2. The front face of each deformable lens is protected by a rigid plastic cover. Pumps containing the liquid that will fill the lens are attached to each side of the spectacle frame. The linear power-volume relationship coupled with suitably sized and positioned adjustment dials make the process of self-optimisation smooth and natural. When the user is happy that he (or she) has obtained a sharp image and optimised his (or her) refraction each lens is then sealed by tightening a small key. The adjustment mechanisms are then removed to leave a light-weight (the current device weighs approximately $50 \mathrm{~g}$ but can be reduced in weight to around $30 \mathrm{~g}$ ) pair of fixed power spectacles. The adjustment mechanism can be left intact if the user requires a variable power low-vision reading aid.

Spectacles using liquid-filled lenses quite different from the Adspecs have been reported before (Bennett, 1973; Pateras et al., 1993), but so far as we are aware, no such spectacles have been applied successfully as a means of vision correction over such a large and hence useful power range prior to the work reported here.

The projected cost of goods of adaptive spectacles after a full development cycle (A. Gupta, personal communication) is approximately $0.5 \mathrm{USD}$, comparable with if not indeed a little lower than the cost of inexpensive spectacles with lenses of the same optical quality. The adaptive spectacles have further cost advantages: their mode of application does not require highly trained and hence expensive personnel to apply and there is the reduction in the need to carry a large inventory as a single adaptive lens may be set to a very large number (strictly an infinite number if quantum fluctuations are ignored) of prescriptions.

\section{Methods}

A preliminary field trial of the effectiveness of the Adspec lens as a means of vision correction has been performed (Afenyo and Silver, 2001). A total of 25 subjects were tested and it was shown that it is indeed possible to obtain good vision correction using self-adjusted Adspecs. We now present the details and results of a new and much more extensive field trial of the Adspec lens.

The experiments were performed in South Africa, Ghana, Malawi and Nepal and the results collated. A total of 213 participants between the ages of 18 and 60 were selected by agencies in each country and communicated with through an interpreter. Distance visual acuity was measured with either a standard Snellen chart or an illiterate E-chart positioned at $6 \mathrm{~m}$. Because of the nature of the location some of the eye tests were performed outside in daylight and so illumination was subject to variation.

The unaided vision data for each subject was recorded for each eye while occluding the fellow eye. For those subjects who could not read an entire line, the number of unread letters, $\mathrm{N}$, on that line was also recorded. Then, using the conventional trial frame method, an optometrist determined the refraction for each subject, recording the spherical and cylindrical correction for each eye. The vision test procedure was repeated with the same chart to obtain the subjects' acuity using trial lenses.

The trial lenses were removed and the subject was asked to relax their eyes by looking at a distant target. A visual target was chosen at a distance greater than $6 \mathrm{~m}$ and the subject was then asked to wear the Adspecs and to carry out the following adjustment protocol. Both left and right Adspec lenses were initially set to $+6 \mathrm{D}$ before they were worn to provide sufficient fogging to eliminate unwanted accommodation. The subjects' left eye was occluded and the subject then asked to adjust the right lens, slowly decreasing the power until the target came into sharp focus. The right eye was then occluded and the left eye revealed. The subject was subsequently asked to adjust the left lens in the same manner until the target was again in focus. Then, when viewing the target binocularly, the subject was asked to adjust the power and go slightly past the point of sharpest focus until the image began to blur and then turn the dial backwards slightly to achieve the sharp focus again. This is based on our observation that best acuity could be achieved if a final fine adjustment was made binocularly, i.e. when the vergence system functions. Following this, the subject was given an acuity test using the same chart as in the preceding tests. The binocular acuity obtained whilst using the Adspecs was recorded. The Adspecs were then removed and the spherical power of each lens was determined using either a Pentax OLH-10 (Pentax Corporation, Tokyo, Japan) or a Topcon (Topcon Corporation, Tokyo, Japan) focimeter.

\section{Results}

To compare the self-optimised refractive power obtained using the Adspec lens with the optometrist 


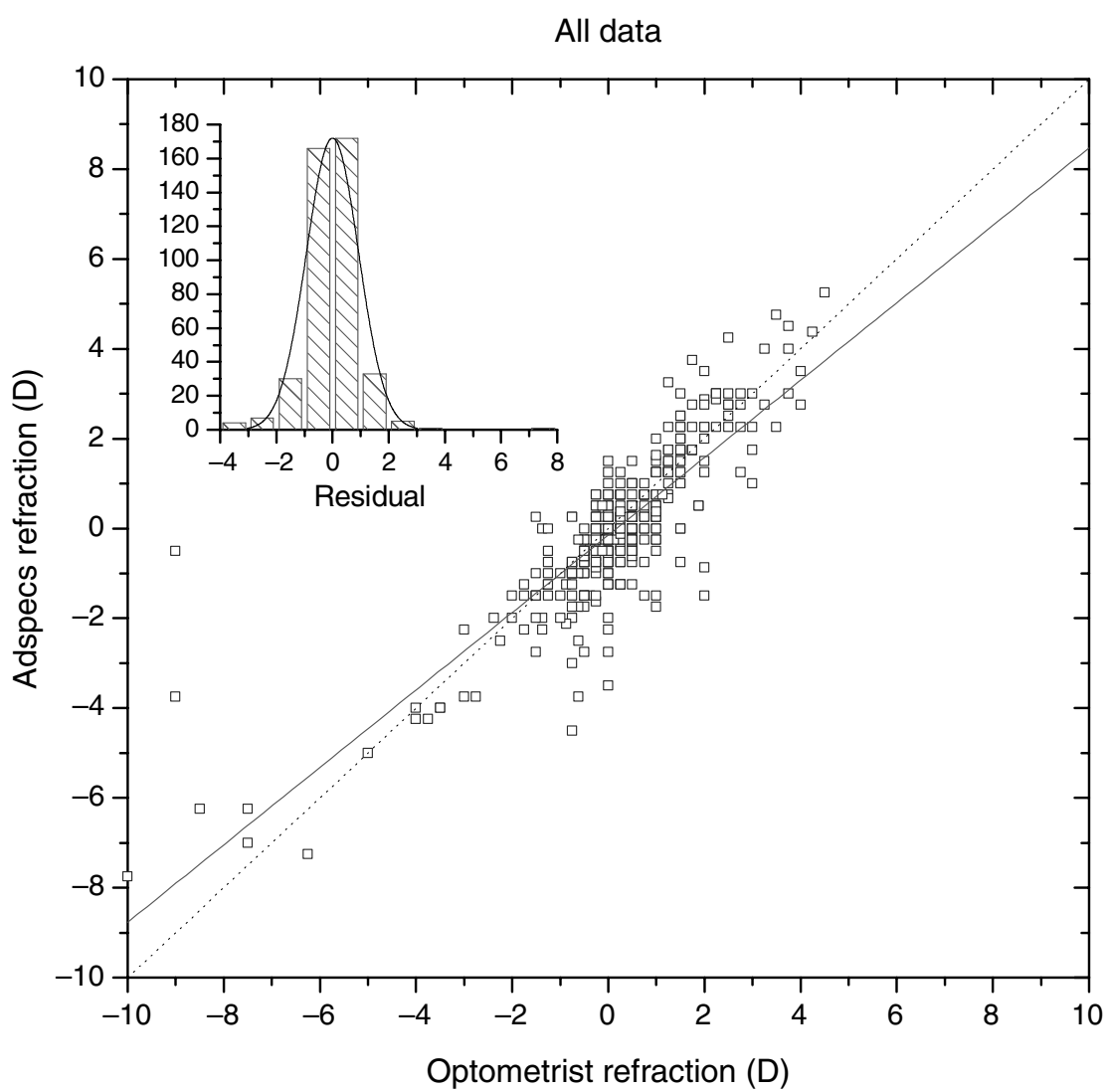

Figure 3. Refraction data for the complete set of eyes tested in the field trial. The Adspecs self-refraction (D) is plotted against the optometrist refraction (D). Also shown is the line $y=x$ (broken) and the least squares linear regression line $y=(-0.148 \pm 0.046)+(0.826 \pm 0.0026) x$. Correlation coefficient $r=0.84$. Inset shows histogram of residuals and fitted normal curve.

refraction one must take account of the fact that the Adspec lenses used in the trial are spherical. Hence, with these lenses no attempt was made to correct cylindrical astigmatism. The optometrist refraction measurements were expressed as an equivalent spherical power, $S_{\mathrm{E}}$, where $S_{\mathrm{E}}=S+C / 2$. Figure 3 shows the Adspecs selfoptimised refraction (D) against the optometrist spherical equivalent refraction (D). The line $y=x$ (shown broken) has been included on this graph: if the selfadjustment procedure agreed exactly with the optometrist prescription in every case, all the points would coincide with this line. Some of the outlying data points are clearly erroneous and have been omitted from subsequent work; this arose because some subjects who took part in the trials were found to have pathology and were later referred to an ophthalmic hospital. The histogram of the residual distribution, [Adspecs (D) - optometrist (D)], was also plotted and is shown in the inset. From Figure 3, it is seen that all but seven points $(1.8 \%)$ lie within the optometrist refraction range $(-5$ to $+5 \mathrm{D})$ with a larger proportion between $(-1 \mathrm{D}$ and $+1 \mathrm{D})$. The linear regression line has slope 0.826 with an error of 0.026 . The correlation coefficient for the complete data set is $r=0.84$ showing a good linear relationship. The corresponding residual histogram is strongly peaked around the centre but slightly asymmetric tending towards negative residual values; this is because hypermetropes tend to select a less positive Adspec power and myopes tend to select a more negative Adspec power.

We now look at the data for just those subjects who suffer from little or no astigmatism. If we remove those subjects requiring greater than $\pm 0.5 \mathrm{D}$ cylinder correction we arrive at the 'non-astigmatic' data plotted in Figure 4. In this case the linear regression line has a slope of 1.00 with an error of 0.004 showing that the slope is not significantly different from 1 . The correlation coefficient is found to be $r=0.82$, again showing a good linear relationship.

It is clear from Figures 3 and 4 that those subjects requiring little or no correction can show a tendency to 'over-minus' their Adspec refraction. This suggests that accommodation has been stimulated with the subject still maintaining a sharp image. This demonstrates the importance of using a correct protocol, i.e. one that minimises accommodation by fogging.

We now compare the MAR distributions. Figure 5 is a histogram showing the unaided MAR distribution of the 


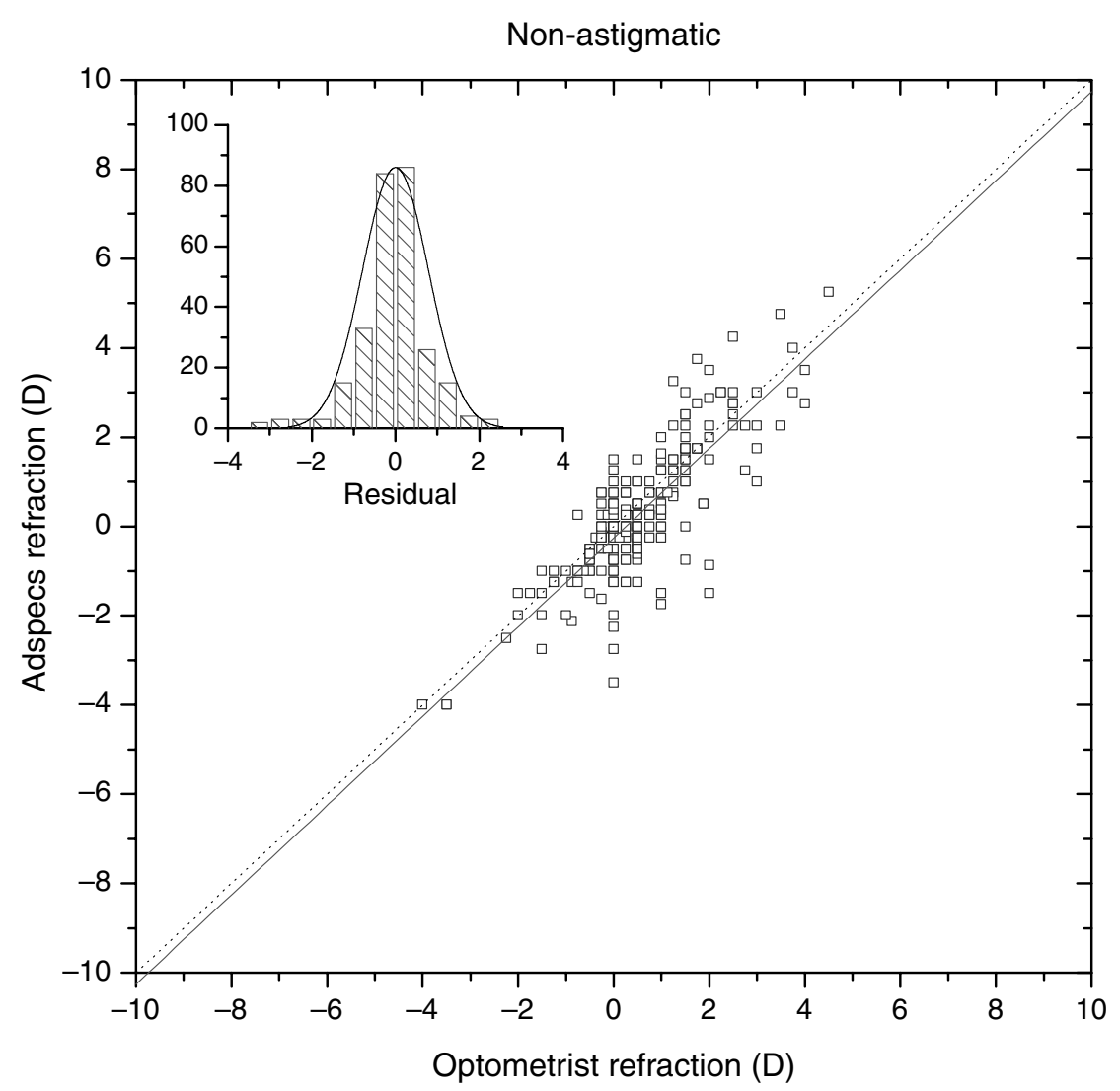

Figure 4. Refraction data for the set of non-astigmatic eyes tested in the field trial. Adspecs self-refraction (D) is plotted against optometrist refraction (D). Also shown is the line $y=x$ (broken) and the least squares linear regression line $y=(-0.257 \pm 0.051)+(1.00 \pm 0.004) x$. Correlation coefficient $r=0.82$. Inset shows histogram of residuals and fitted normal curve.

trial participants. Figure 6 shows the MAR obtained after self-correction with the Adspecs. Where a subject had little or no vision in one eye the monocular MAR of their good eye has been included. It is seen from the unaided MAR distribution that the great majority, some $78 \%$, of this sample cannot obtain $6 / 6$ vision and therefore require some sort of vision correction. After the self-adjustment procedure with the Adspecs just $13 \%$ of the sample had acuity worse than $6 / 9$. So, by the simple use of the Adspecs, $87 \%$ of this sample population could obtain eyesight of more than sufficient quality to satisfy the current Driver and Vehicle Licensing Agency (DVLA) ${ }^{2}$ minimum vision requirement for a driver of a lorry or a bus.

\footnotetext{
${ }^{2}$ In the UK the DVLA (http://www.dvla.gov.uk) currently require that new drivers of group 1 vehicles (motor cars and motor cycles) can read, in good light, a registration mark fixed to a motor vehicle and containing letters and figures $79 \mathrm{~mm}$ high and $50 \mathrm{~mm}$ wide at a distance of $20 \mathrm{~m}$. This corresponds to a binocular acuity of approximately 6/12. Whereas drivers of group 2 vehicles (lorries and buses) are required to have an acuity that is at least $6 / 9$ on the Snellen scale in the better eye and 6/12 on the Snellen scale in the other eye.
}

\section{Conclusion}

We have described the Adspecs and have shown that, provided the correct fogging protocol is used, the Adspec liquid-filled lens is a good method of both determining and correcting refractive error. The comparisons between the self-optimised Adspec refractions and the optometrist refractions obtained by standard means demonstrates the success of this procedure. This conclusion is strengthened if account is made of the uncertainty inherent in current methods of automated and clinical refraction (Bullimore et al., 1998).

The efficacy of this method in practice and the benefit that Adspecs can provide in real terms has also been illustrated using a comparison of the MAR distributions. This type of vision improvement can have a very significant and immediate impact on the lives of people from developing nations who would otherwise be excluded from education or employment. A separate study performed in 2001 of an adult literacy class in Ghana showed that amongst the adult learners who had dropped out of their class, $93 \%$ were found to need vision correction. The Adspecs were well received by 


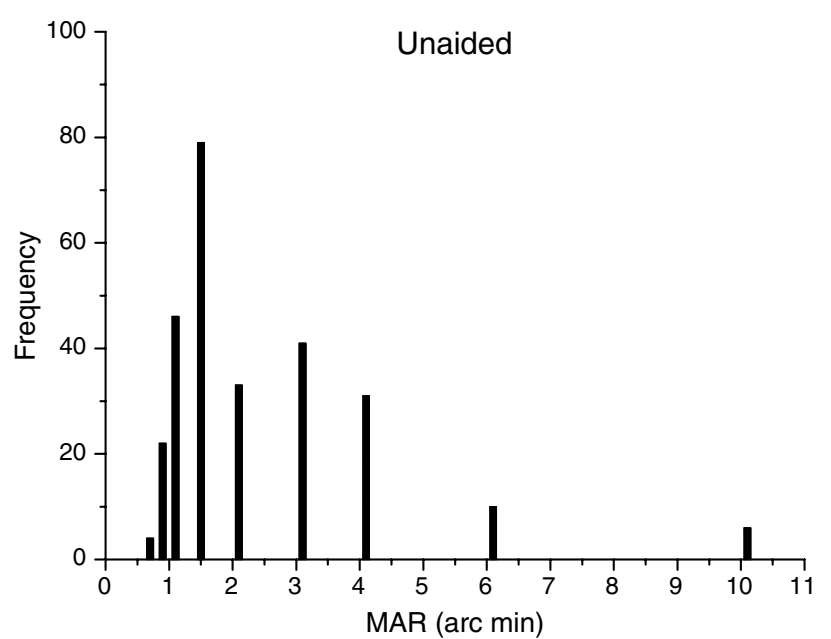

Figure 5. Unaided Minimum Angle of Resolution (arc min). Histogram showing the distribution of MAR for all subjects in the field trial before self-optimised correction.

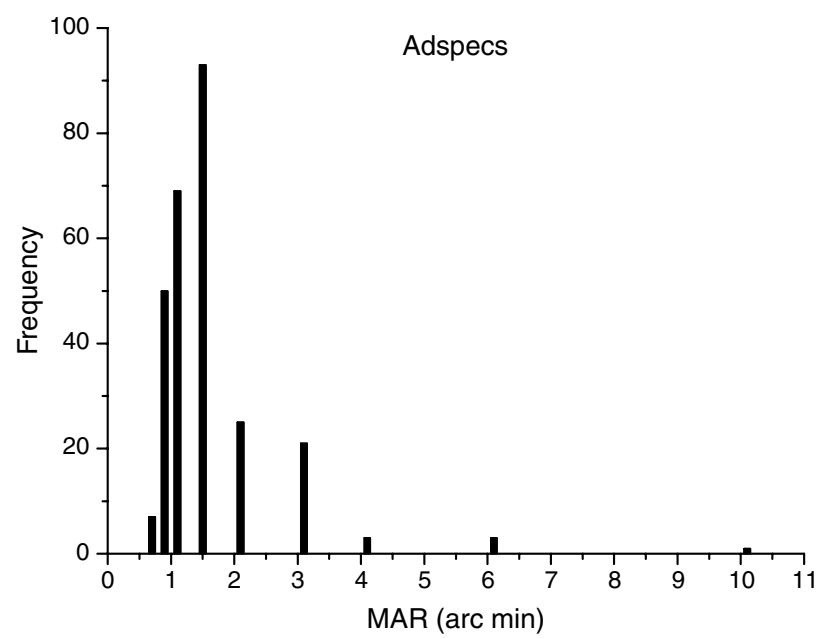

Figure 6. Adspecs Minimum Angle of Resolution (arc min). Histogram showing the distribution of MAR for all subjects in the field trial after self-optimised correction with the Adspecs.

these students who found them comfortable to wear and expressed the wish to continue using them. Another trial in much harsher working conditions was also performed; in Madurai, India, a group of manual workers in a cotton-spinning factory wore the Adspecs over a period of 5 weeks during which their productivity was monitored. The workers found the Adspecs to be effective and practicable. The Adspecs are currently worn by one of the authors as a replacement for his conventional spectacles and over a period of 4 years use they have been found to be durable, robust and sturdy.

The Adspecs not only provide useful corrective eye wear but also a means of effectively determining one's own refraction. Taken together, one can conclude that the Adspecs can provide a possible solution to the problem faced by approximately 1 billion people in the developing world currently without the vision correction they require.

\section{Discussion}

The Adspecs is one solution to the problem of supplying vision correction to those in underdeveloped countries. They are expected to prove particularly attractive in terms of cost, logistics, speed and ease of application when compared with the alternative of supplying a practitioner with a refractometer and a selection of graduated lenses (A. Gupta, personal communication).

The present study includes only adults. There are two reasons for this: first an understanding of the protocol is needed, this is difficult enough with a language and social barrier, and secondly, a child's eye has a vastly increased range of accommodation. The problem of determining the correct refraction for children needs further investigation. But given the intuitive means by which the Adspecs can be adjusted to obtain clear vision they might aid a solution if supervised by someone with an appropriate level of training.

There are two components to the Adspec method, self-optimisation and continuous variable focus. Further research might indicate that a continuous mode of adjustment may facilitate more reliable refractions than currently available using standard subjective clinical refraction techniques where the $95 \%$ limits of agreement are between -0.90 and $+0.65 \mathrm{D}$ (Bullimore et al., 1998). Compound this error with trial lenses graduated in $0.25 \mathrm{D}$ steps and the advantages of a continuous method are reinforced.

A new study of the use of Adspecs in the rehabilitation of low vision conditions in the UK is already underway.

\section{Acknowledgements}

The authors wish to thank the UK Government's Department for International Development (DFID) for their support in this project and for providing partial funding for travel and subsistence for our field work in Africa and Asia. We would also like to thank G. Afenyo, A.S. Carlson, J. Coughlin, S. Gardiner, and L. Jenkin for their role in collecting data and A. AddoMensah, K. Griffin and M. Wills for help with the planning and administration of the trials and D. Crosby for his technical assistance with statistical software and his rendered diagram of the Adspecs.

\section{References}

Afenyo, G. D. and Silver, J. D. (2001) Vision Correction with Adaptive Spectacles World Blindness and its Prevention, Vol. 
6. International Agency for the Prevention of Blindness, Hyderabad, India.

Bennett, A. G. (1973) Variable and progressive power lenses. Manufact. Opt. Int. 26, 88-91.

Bennett, A. G. and Rabbetts, R. B. (1998) Clinical Visual Optics, 3rd edn. Butterworth Heinemann, London. Table 21.2, p. 407.

Bullimore, M. A., Fusaro, R. E. and Adams, C. W. (1998) The repeatability of automated and clinical refraction. Optom. Vis. Sci. 75, 617-622.

Dandona, R., Dandona, L., Srinivas, M., Sahare, P., Narsaiah, S., Munoz, S. R., Pokharel, G. P. and Ellwein, L. B. (2002) Refractive error in children in a rural population in India. Invest. Ophthalmol. Vis. Sci. 43, 615-622.

Guirao, A., Porter, J., Williams, D. R. and Cox, I. G. (2002) Calculated impact of higher-order monochromatic aberrations on retinal image quality in a population of human eyes. J. Opt. Soc. Am. A 19, 1-9.

Jenkins, F. A. and White, H. A. (1976) Fundamentals of Optics, 4th edn. McGraw-Hill International Editions, New York. Chapter 4, p. 67.

Maul, E., Barroso, S., Munoz, S. A., Sperduto, R. D. and Ellwein, L. B. (2000) Refractive error study in children: results from La Florida, Chile. Am. J. Ophthalmol. 129, 445-454.

Pateras, E. S., Fowler, C. W. and Chandrinos, A. B. (1993) Deformable spectacle lenses. Ophthal. Physiol. Opt. 13, 97-99.

Porter, J., Guirao, A., Cox, I. G. and Williams, D. R. (2001) Monochromatic aberrations of the human eye in a large population. J. Opt. Soc. Am. A. 18, 1793-1803.

Sorsby, A., Sheridan, M. and Leary, G. A. (1960) Vision, visual acuity, and ocular refraction of young men. Br. Med. J. 1, 1394-1398.

Sperduto, R. D., Seigel, D., Roberts, J. and Rowland, M. (1983) Prevalence of Myopia in the United States. Arch. Ophthalmol. 101, 405-407.

Stenström, S. (1946). Untersuchungen über die Variation undKovariation der optischen E; emente des menshlichen Auges. Acta Ophthal., Suppl. 26. (English translation by Woolf, D., Am. J. Optom. 25, 218-232, 1998)

Strömberg, E. (1936) Ueber Refraktion und Achsenlänge des menschilichen Auges. Acta Ophthal. 14, 281-293.

Sugiura, N. and Morita, S. (1993) Variable focus liquid filled optical lens. Appl. Opt. 32, 4181-4186.

World Health Organization (1987) The Provision of Spectacles at Low Cost. WHO, Geneva.

Zhao, J., Pan, X., Sui, R., Munoz, S. R., Sperduto, R. D. and Ellwein, L. B. (2000) Refractive error study in children: results from Shunyi District, China. Am. J. Ophthalmol. 129, $427-435$

\section{Appendix A}

\section{Derivation of lens formulae}

From the classical theories of fluid dynamics and elasticity one can derive the equation that describes the surface of the lens. One needs to describe the deformation of an elastic surface produced by a liquid under pressure.
The standard approach is to neglect the effects of gravity and equate the elastic forces produced by the tension across the deformed elastic surface to the force supplied by the liquid under pressure across a plane perpendicular to the optical axis. If the film is thin, the absolute tension in the film is the same at all points. With the surface boundary fixed so that its displacement is zero at all points on a circular perimeter of radius $a$, the surface becomes a paraboloid (Sugiura and Morita, 1993):

$z=\frac{w}{4 T}\left(a^{2}-x^{2}-y^{2}\right)$

where $w$ is the liquid pressure and $T$ is the tension per unit length in the elastic film. Over the central area the surface is a sphere with radius $w / 2 T$.

We can now derive the spherical power-volume relationship directly using a geometrical approach. Consider a sphere of radius $R$ now centred on the origin of Cartesian coordinates $(x, y, z)$ as shown in Figure A1,

$x^{2}+y^{2}+z^{2}=R^{2}$.

A cap of this sphere of height $h$ has volume $V$ given by

$V=\int_{R-h}^{R} \pi x^{2} \mathrm{~d} z=\pi R h^{2}+\mathrm{O}\left(h^{3}\right)$,

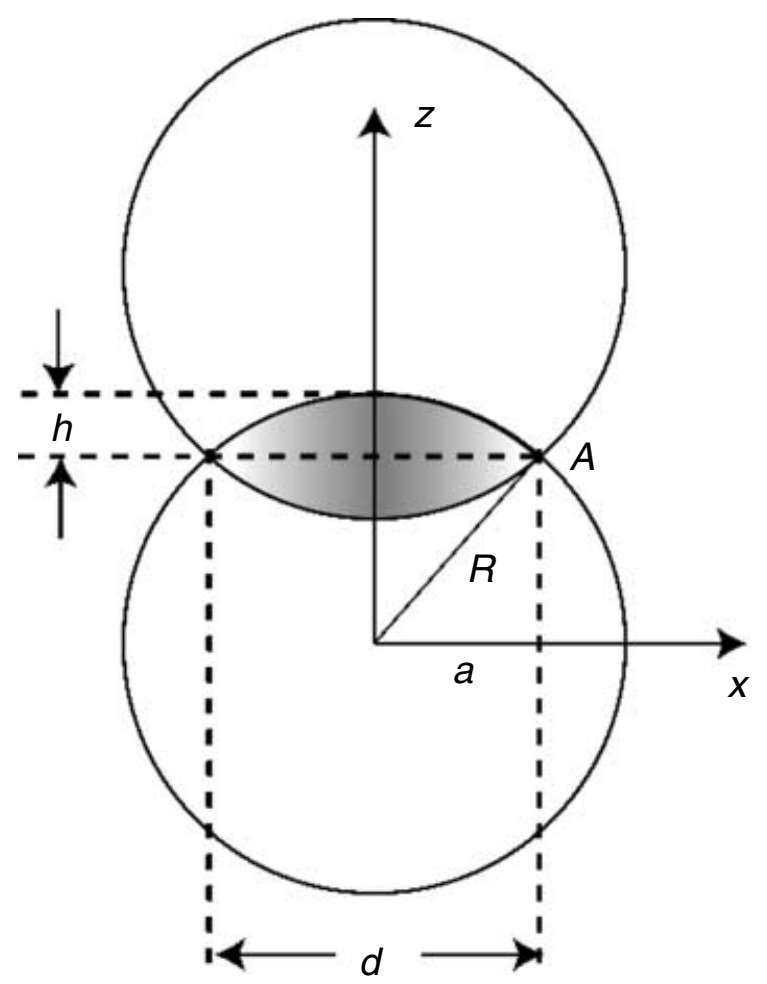

Figure A1. The deformation of the elastic membrane produces a parabolic surface. Over a region near the optical $(z)$ axis where the deflection, $h$, is small compared with the radius of curvature, $R$, the surface is spherical. 
where, because the deformation of the lens surface is small compared with the radius of curvature of the lens, $h=R$. The Adspecs lens can be thought of as two identical spherical caps or plano-convex lenses placed back to back. Thus the lens volume is $V_{\mathrm{L}}=2 \mathrm{~V}$, and so from Equation (A3),

$R=\frac{V_{\mathrm{L}}}{2 \pi h^{2}}$.

At point A on the diagram where $x=d / 2, y=0$ and $z=R-h$, we can write, using Equation (A2),

$h^{2}-2 R h+\frac{d^{2}}{4}=0$,

where $d$ is the diameter of the circular lens frame. Solving Equations (A4) and (A5) for $R$ and neglecting terms in $h^{3}$ and smaller, we find that the radius of curvature of the lens is given by
$R=\frac{\pi d^{4}}{32 V_{\mathrm{L}}}$

The refractive power of a bi-convex lens is given by the formula (Jenkins and White, 1976):

$P=\frac{2\left(n_{\mathrm{L}}-1\right)}{R}$,

where $n_{\mathrm{L}}$ is the refractive index of the lens material, hence, from Equation (A6):

$P=\frac{64\left(n_{\mathrm{L}}-1\right) V_{\mathrm{L}}}{\pi d^{4}}$.

In the actual Adspecs frame construction there is a small residual cylindrical volume between the two caps and so for the Adspecs lens:

$P_{\text {Adspecs }}=\frac{64\left(n_{\mathrm{L}}-1\right)}{\pi d^{4}}\left(V-V_{0}\right)$. 